\title{
Seasonal evaluation of the effluent quality of the Rosarito, Mexico wastewater treatment plant and identification of alternatives for its indirect reuse
}

\author{
A. E. O. Leal, J. G. V. Rodríguez, E. L. Velez, L. A. A. Hurtado \\ \& E. L. Leal \\ Facultad de Ciencias Químicas e Ingeniería. UABC, México
}

\begin{abstract}
This project addressed the problem of reusing treated wastewater, which is a common practice in the European Union, the United States, Canada, and some parts of Africa. The reuse of treated wastewater is not common in Mexico, and the majority of such water is discharged into the ocean and, to a lesser extent, on land. Because the reuse of this resource depends to a large extent on the quality of the treatment plant discharge, in this study we attempted to evaluate seasonal fluctuations in the quality of the Rosarito Norte treatment facility effluent over the course of one year. The majority of the total dissolved solids were found to consist of chlorides and sulfates such as sodium salts. Also reflected in the overall level of water hardness were salts derived from calcium and magnesium, as well as the carbonates and bicarbonates. These salts were not evaluated in this part of the project, but deserve consideration. The quantity of organic material was minimal with respect to $\mathrm{BOD}_{5}$ (not up to $270 \mathrm{mg} / \mathrm{L}$ ), and did not present a problem for the reuse of treated wastewater. The majority of the nitrogen present (TKN: $1.9-86.3 \mathrm{mg} / \mathrm{L}$ ) was found to consist of proteins that remained as a result of the short curing time, as well as the poor oxygenation during the treatment process. Also present were high levels of total phosphorus and sulfates, between $15.5-38.2 \mathrm{mg} / \mathrm{L}$ and $197-1200 \mathrm{mg} / \mathrm{L}$, respectively. The microbiological and parasitic analyses yielded excellent results, testing positive in only $33 \%$ and $0 \%$ of the trials, respectively. These results were compared with data reported for the aquifer into which the water will be infiltrated, and will be shared with the Tijuana office of the State Commission of Services for use as a basis for making decisions regarding the reuse of treated potable water.
\end{abstract}

Keywords: wastewater, reuse, indirect reuse. 


\section{Introduction}

Although the reuse of treated wastewater is still relatively rare in Mexico, with the majority of such water being discharged into the ocean and to a lesser extent on land [1], the problem of reusing this resource has become increasingly urgent in recent decades.

It is important to take advantage of the fact that the Northwest region of the country, particularly Tijuana and Playas de Rosarito Mexico, has sewer system coverage of slightly over $80 \%$. In addition to its current use for irrigation, the collected wastewater could also be used to indirectly recharge aquifers, thereby augmenting the supply of potable water available to the region [2].

This practice is used the US, in states including California, New Jersey, and Georgia, where the underground water supplies are replenished with treated wastewater. In the coastal region of California's Orange County, treated wastewater is injected into the subsoil to help prevent the local aquifer from becoming contaminated by saltwater.

The county of Los Angeles has been using surface dispersion to recharge local aquifers since the 1960s. The city of Perth Amboy in New Jersey maintains two open reservoirs to replenish the local aquifer and to help protect it from the intrusion of salt water. The state of Georgia's Clayton County applies uses recycled wastewater to its watershed [3].

It is clear that the indirect reuse of treated wastewater is a viable alternative for helping to guarantee an adequate water supply to our region, and this option is included in the Plan Maestro de Agua Potable y Saneamiento de Tijuana y Playas de Rosarito 2003 [4], the document describing the plan of the State Commission of Public Services to provide water to the cities of Tijuana and Playas de Rosarito.

One of the main objections to the reuse of treated wastewater is the lack of sufficient information regarding the current quality of the wastewater treatment plant effluent.

The present study addresses this issue by providing a seasonal evaluation of the physicochemical, organic, and biological properties of the Rosarito Norte wastewater treatment plant effluent for the purpose of identifying alternatives for indirect reuse.

\section{Site of the study}

Considering that the quality of the water treatment plant effluent is the factor limiting its reuse, it is necessary to determine the seasonal variation in the quality of the effluent originating from the treatment plants thought to be most efficient, as is the case with the Planta de Tratamiento de Agua Residual de Rosarito Norte (PTARRN), a wastewater treatment facility located in city of Playas de Rosarito at $12^{\circ} 22^{\prime} 21^{\prime \prime} \mathrm{N}$ and $116^{\circ} 54^{\prime} 15^{\prime \prime}$ (see figure 1 [5]). 

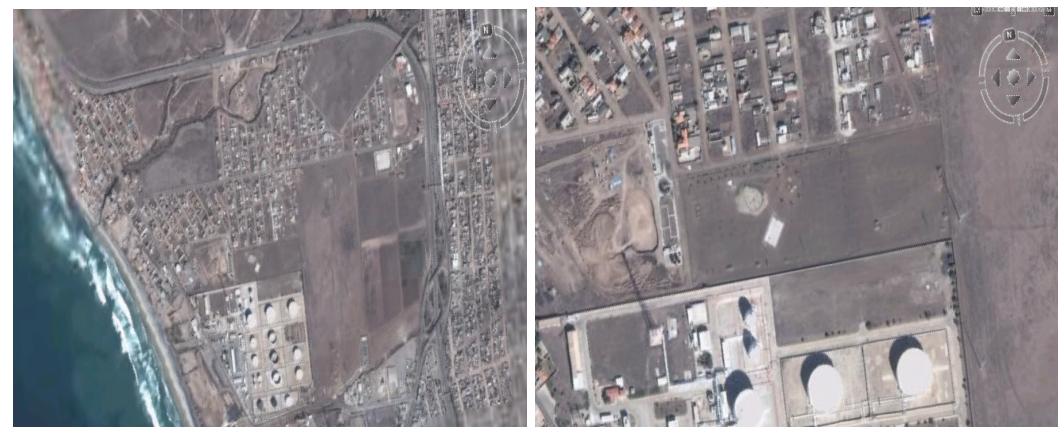

Figure 1: $\quad$ Site of the study.

\section{Materials and methods}

The collection and preservation of the water samples were carried out by personnel of the State Commission of Public Services of Tijuana in accordance with the government standards outlined in NMX-AA-003 [6].

The laboratory work consisted of an analysis of 36 samples composed of effluent taken from the North Rosarito plant (PTARRN) during the period from November 2006 to December 2007. The analyses performed on each sample included six physical, six microbiological, and 20 chemical. Each of these analyses was carried out and replicated at ISO 9001:2000 certified laboratories at the University of Baja California's Tijuana campus.

The analysis of 25 pesticides, 55 volatile, and 16 semivolatile organic compounds were carried out using methods established by the US Environmental Protection Agency [7]. An analysis of eight trace metals was done at the CESPT wastewater treatment plant at Punta Bandera, Tijuana.

A statistical analysis of the laboratory results including average values, total and seasonal variances, and standard deviation was done using E-Views software.

Equipment used included a Hach DR-4000 UV-VIS spectrophotometer, Finnigan CG Ultratrace coupled with an MS Finnigan Polaris Q with ion trap. Metal quantification was done with a Varian Ultramass 700 ICP-MS, using the US American Public Health Association's method 3000 (APHA 1999). A conventional microscope and a BBL Crystal system were used to identify helminth eggs and bacteria, respectively.

Most sampling was done weekly, with samples being taken on each of the seven days of the week.

\section{Results}

As can be seen in Table 1, the majority of the total dissolved solids was found to consist of chlorides and sulfates such as sodium salts. Also reflected in the 
overall level of water hardness were salts derived from calcium and magnesium, as well as carbonates and bicarbonates. These salts were not evaluated in this part of the project, but deserve consideration.

Table 1: $\quad$ Results of chemiophysical analysis.

\begin{tabular}{||c|c|c|c|c|c|c||}
\hline Parameter & $\begin{array}{c}\text { Minimum } \\
\text { value }\end{array}$ & $\begin{array}{c}\text { Maximum } \\
\text { value }\end{array}$ & Average & $\begin{array}{c}\text { Standard } \\
\text { Deviation }\end{array}$ & Variance & $\begin{array}{c}\text { Number of } \\
\text { samples }\end{array}$ \\
\hline \hline pH & 6.45 & 7.7 & 7.43 & 0.2696 & 0.072 & 36 \\
\hline Conductivity & 1630 & 2240 & 2050.28 & 131.29 & 17238.15 & 35 \\
\hline Color & 40 & 255 & 73.13 & 41.06 & 1686 & 36 \\
\hline Turbidity & $<14$ & 25 & 19.2 & 3.89 & 15.2 & $5^{*}$ \\
\hline TDS & 1100 & 2115 & 1375.73 & 199.36 & 39744.5 & $34^{* *}$ \\
\hline TSS & $<10$ & 138 & 57.5 & 54.28 & 2947 & $4^{*}$ \\
\hline Total Hardness & 110 & 1394 & 503.91 & 195.39 & 38180.02 & 36 \\
\hline Chloride & 140 & 362 & 286.44 & 40.669 & 1653.968 & 36 \\
\hline Fluoride & 0.09 & 2.2 & 1.22 & 0.517 & 0.268 & $35^{* *}$ \\
\hline Phenols & $<0.001$ & 0.006 & 0.0029 & 0.0016 & $2.59 \mathrm{E}-06$ & $21^{*}$ \\
\hline Surfactants & 0.03 & 1 & 0.198 & 0.19 & 0.036 & $35^{* *}$ \\
\hline Total Chlorine & $<0.01$ & 2.2 & 0.177 & 0.381 & 0.145 & $31^{*}$ \\
\hline Free Chlorine & $<0.02$ & 1.6 & 0.131 & 0.303 & 0.091 & $26^{*}$ \\
\hline Cyanide & $<0.01$ & 0.2 & 0.028 & 0.033 & 0.001 & $31^{*}$ \\
\hline Nitrate & 0.01 & 3 & 0.446 & 0.708 & 0.502 & 36 \\
\hline Nitrite & $<0.001$ & 2.5 & 0.202 & 0.441 & 0.194 & $33^{*}$ \\
\hline Ammonia & 0.25 & 110.8 & 13.88 & 21.9 & 479.62 & 36 \\
\hline TKN & 1.9 & 86.3 & 29.19 & 20.88 & 436.01 & 36 \\
\hline Orthophosphate & 2.4 & 33.6 & 13.61 & 10.35 & 107.2 & 36 \\
\hline Total Phosphate & 15.5 & 38.2 & 28.8 & 5.85 & 34.31 & $29^{* *}$ \\
\hline Sulfates & 197 & 1200 & 461.3 & 197.15 & 38869.7 & 36 \\
\hline Oil and Greases & $<5$ & 270 & 63.38 & 67.69 & 4583.075 & $18^{*}$ \\
\hline BOD & $<5$ & 41 & 16.5 & 16.46 & 271 & $44^{*}$ \\
\hline COD & 30 & 363 & 69.75 & 55.44 & 3074.02 & 36 \\
\hline Note: *Samples falling below detectable levels are not included & & & \\
**Number includes only samples that could be analyzed & & & & & \\
\hline
\end{tabular}

The quantity of organic material was minimal with respect to $\mathrm{DBO}_{5}$, and does not represent a problem for the reuse of treated wastewater.

The majority of the nitrogen present (TKN: 1.9-86.3 $\mathrm{mg} / \mathrm{L}$ ) was found to consist of proteins that remained as a result of the short retention time, as well as the poor oxygenation during the treatment process.

Table 2 reveals that the analyses of microbiological contaminants yielded positive results in only $33 \%$ of the cases. Table 3 shows that the tests for helminth eggs were all negative, with only one sample testing positive for uncinaria larvae.

None of the 16 samples analyzed for trace metals exceeded the limits established in Mexican government standard NOM-001-ECOL-1996 [8] for each of the eight metals mentioned in the standard.

As shown in table 5 , only 11 of the 36 samples analyzed showed one or more of the 17 different volatile compounds detected.

Table 6 shows the comparison of parameters having the highest values or fluctuations with regard to the limits established in relevant Mexican federal standards for reservoirs and regional water supplies. 
Table 2: $\quad$ Results of microbiological analysis.

\begin{tabular}{|c|c|c|c|c|c|c|}
\hline Sample & $\begin{array}{l}\text { Total Coliforms } \\
\text { NMP/100ml }\end{array}$ & $\begin{array}{l}\text { Fecal Coliforms } \\
\text { NMP/100ml }\end{array}$ & $\begin{array}{c}\text { Mold } \\
\text { UFC/ml }\end{array}$ & $\begin{array}{c}\text { Yeast } \\
\text { UFC/ml }\end{array}$ & Enterococcus & Enterobacterias \\
\hline 09Nov06 & Negative & Negative & Negative & Negative & Negative & Negative \\
\hline $22 \mathrm{Nov} 06$ & Negative & Negative & Negative & Negative & Negative & Negative \\
\hline 17Jan07 & Negative & Negative & Negative & Negative & Negative & Negative \\
\hline $08 \mathrm{Feb} 07$ & Negative & Negative & Negative & Negative & Negative & Negative \\
\hline $28 \mathrm{Feb} 07$ & Negative & Negative & Negative & Negative & Negative & $\begin{array}{c}\text { Pseudomona } \\
\text { Aeroginosa }\end{array}$ \\
\hline 06Mar07 & Negative & Negative & Negative & Negative & Negative & Negative \\
\hline 14Mar07 & Negative & Negative & Negative & Negative & Negative & Negative \\
\hline $22 \mathrm{Mar} 07$ & Negative & Negative & Negative & Negative & Negative & Negative \\
\hline 10Apr07 & Negative & Negative & Negative & Negative & Negative & Negative \\
\hline $10 \mathrm{Apr} 07$ & Negative & Negative & Negative & Negative & Negative & Negative \\
\hline $14 \mathrm{Apr} 07$ & Negative & Negative & Negative & Negative & Negative & Negative \\
\hline $22 \mathrm{Apr} 07$ & 3 & 3 & 2 & 1 & Negative & E. coli \\
\hline 01Jun07 & Negative & Negative & Negative & Negative & Negative & Negative \\
\hline 09Jun07 & Negative & Negative & Negative & 2 & Negative & Negative \\
\hline $13 \mathrm{Sep} 07$ & Negative & Negative & Negative & Negative & Negative & Enterobacter cloacae \\
\hline $20 \mathrm{Sep} 07$ & Uncountable & Uncountable & Negative & Negative & $\begin{array}{c}\text { Enterococcus } \\
\text { faecium }\end{array}$ & Negative \\
\hline 21 Sep07 & Negative & Negative & Negative & Negative & Negative & Enterobacter cloacae \\
\hline 29Sep07 & Negative & Negative & Negative & Negative & $\begin{array}{l}\text { Staphylococcus } \\
\text { hominis }\end{array}$ & Negative \\
\hline 070ct07 & 30 & Negative & Negative & Negative & Negative & Negative \\
\hline $15 \mathrm{Oct} 07$ & 15 & Negative & Negative & Negative & Negative & Negative \\
\hline $300 \mathrm{ct} 07$ & Negative & Negative & Negative & Negative & Negative & Enterobacter cloacae \\
\hline
\end{tabular}

Table 3: $\quad$ Results of analysis of helminth eggs.

\begin{tabular}{|c|c|}
\hline Number of Sample & Results \\
\hline 1 & "Negative \\
\hline 2 & Negative \\
\hline 3 & Negative \\
\hline 4 & Negative \\
\hline 5 & Negative \\
\hline 6 & Negative \\
\hline 7 & Negative \\
\hline 8 & Negative \\
\hline 9 & Uncinaria Larvae \\
\hline 10 & Negative \\
\hline 11 & Negative \\
\hline 12 & Negative \\
\hline 13 & Negative \\
\hline 14 & Negative \\
\hline 15 & Negative \\
\hline 16 & Negative \\
\hline 17 & Negative \\
\hline 18 & Negative \\
\hline 19 & Negative \\
\hline 20 & Negative \\
\hline 21 & Negative \\
\hline 22 & Negative \\
\hline 23 & Negative \\
\hline
\end{tabular}


Table 4: $\quad$ Results of analysis of metals.

\begin{tabular}{||c|c|c|c|c|c|c|c|c||}
\hline \hline \multirow{2}{*}{ Date } & \multicolumn{9}{|c|}{ Analysis (mg/L) } \\
\cline { 2 - 10 } & Arsenic & Cadmium & Copper & Chromium & Mercuric & Nickel & Lead & Zinc \\
\hline \hline 9Nov06 & 0.004 & $<$ LD & 0.007 & 0.001 & $<$ LD & 0.005 & 0.001 & 0.062 \\
\hline 22 Nov06 & $<$ LD & $<$ LD & 0.03 & 0.003 & $<$ LD & 0.05 & $<$ LD & 0.043 \\
\hline 22 Mar07 & $<$ LD & $<$ LD & $<$ LD & $<$ LD & NA & $<$ LD & $<$ LD & 0.087 \\
\hline 30 Mar07 & $<$ LD & $<$ LD & $<$ LD & $<$ LD & NA & $<$ LD & $<$ LD & 0.126 \\
\hline 10 Apr07 & $<$ LD & $<$ LD & 0.008 & $<$ LD & $<$ LD & $<$ LD & $<$ LD & 0.075 \\
\hline 14 Apr07 & $<$ LD & $<$ LD & $<$ LD & $<$ LD & $<$ LD & $<$ LD & $<$ LD & 0.075 \\
\hline 22 Apr07 & $<$ LD & $<$ LD & 0.004 & 0.006 & $<$ LD & 0.009 & $<$ LD & 0.093 \\
\hline 30Apr07 & $<$ LD & $<$ LD & $<$ LD & $<$ LD & $<$ LD & $<$ LD & $<$ LD & 0.087 \\
\hline 8/May07 & $<$ LD & $<$ LD & $<$ LD & $<$ LD & $<$ LD & $<$ LD & $<$ LD & 0.084 \\
\hline 16 May07 & $<$ LD & $<$ LD & $<$ LD & $<$ LD & $<$ LD & $<$ LD & 0.003 & 0.095 \\
\hline 24May07 & $<$ LD & $<$ LD & $<$ LD & $<$ LD & $<$ LD & $<$ LD & $<$ LD & 0.076 \\
\hline 1Jun07 & $<$ LD & $<$ LD & $<$ LD & $<$ LD & $<$ LD & $<$ LD & $<$ LD & 0.063 \\
\hline 9Jun07 & $<$ LD & $<$ LD & $<$ LD & $<$ LD & $<$ LD & $<$ LD & $<$ LD & 0.057 \\
\hline 29Sep07 & $<$ LD & $<$ LD & $<$ LD & $<$ LD & $<$ LD & 0.006 & 0.001 & $<$ LD \\
\hline 31Oct07 & $<$ LD & $<$ LD & 0.007 & 0.02 & $<$ LD & 0.005 & 0.003 & 0.094 \\
\hline 24 Nov07 & $<$ LD & $<$ LD & $<$ LD & 0.004 & $<$ LD & $<$ LD & 0.001 & 0.067 \\
\hline NOM-001 & 0.1 & 0.1 & 4 & 0.5 & 0.005 & 2 & 0.2 & 10 \\
\hline
\end{tabular}

Note: $<\mathrm{LD}=$ Less than detection limit.

Table 5: $\quad$ Results of analysis of organic compounds.

\begin{tabular}{|c|c|c|c|c|c|c|c|c|c|c|c|}
\hline \multirow{2}{*}{$\begin{array}{l}\text { Parameter } \\
\quad(\mathrm{ppm})\end{array}$} & \multicolumn{11}{|c|}{ Sample } \\
\hline & $\begin{array}{l}22 \mathrm{Mar} 0 \\
7\end{array}$ & 17Jun07 & $\begin{array}{l}\text { 04Ago0 } \\
7\end{array}$ & $\begin{array}{l}\text { 20Ago0 } \\
7\end{array}$ & $\begin{array}{l}28 \mathrm{Ago0} \\
7\end{array}$ & 05Sep07 & 13Sep07 & 21Sep07 & 29Sep07 & 10Dec07 & 18Dec07 \\
\hline Chloromethane & 453.6 & N.D. & N.D. & N.D. & N.D. & N.D. & N.D. & N.D. & N.D. & 527.95 & 353.71 \\
\hline Toluene & N.D. & N.D. & 4.4 & 3.91 & N.D. & N.D. & N.D. & N.D. & N.D. & N.D. & N.D. \\
\hline Ethylbenzene & 1.15 & 3.12 & N.D. & N.D. & N.D. & N.D. & N.D. & N.D. & N.D. & N.D. & N.D. \\
\hline $\begin{array}{l}1,1,2,2 \\
\text { Tetrachloroethan } \\
\text { e }\end{array}$ & N.D. & N.D. & 5.98 & N.D. & N.D. & N.D. & N.D. & N.D. & N.D. & N.D. & N.D. \\
\hline $\begin{array}{l}1,3 \\
\text { Dichlorobenzene }\end{array}$ & 4.38 & 3.51 & 1.62 & 0.8 & 3.06 & N.D. & N.D. & N.D. & N.D. & N.D. & N.D. \\
\hline $\begin{array}{l}1,4 \\
\text { Dichlorobenzene }\end{array}$ & 5 & 4.62 & 2.16 & 1.14 & 3.09 & N.D. & N.D. & N.D. & N.D. & N.D. & N.D. \\
\hline $\begin{array}{l}1,2 \\
\text { Dibromoethane }\end{array}$ & N.D. & N.D. & N.D. & N.D. & N.D. & N.D. & N.D. & N.D. & N.D. & N.D. & N.D. \\
\hline $\begin{array}{l}1,2 \\
\text { Diclorobenceno }\end{array}$ & N.D. & 4.61 & 1.57 & 0.98 & 2.95 & N.D. & N.D. & N.D. & N.D. & N.D. & N.D. \\
\hline Acetone & 40 & 71.82 & N.D. & N.D. & N.D. & N.D. & N.D. & N.D. & N.D. & 29.4 & 43.96 \\
\hline 2-Hexanone & N.D. & N.D. & 7.98 & N.D. & N.D. & N.D. & N.D. & N.D. & N.D. & N.D. & N.D. \\
\hline $\begin{array}{l}\text { 4-Methyl-2- } \\
\text { pentanone }\end{array}$ & N.D. & N.D. & 12.47 & N.D. & N.D. & N.D. & N.D. & N.D. & N.D. & N.D. & N.D. \\
\hline $\begin{array}{l}1,2,3- \\
\text { Trichlorobenzen } \\
\mathrm{e}\end{array}$ & N.D. & N.D. & 3.99 & 2.25 & 4.33 & N.D. & N.D. & N.D. & N.D. & N.D. & N.D. \\
\hline $\begin{array}{l}\text { Methyl } \\
\text { methacrylate }\end{array}$ & N.D. & N.D. & 3.13 & N.D. & N.D. & N.D. & N.D. & N.D. & N.D. & N.D. & N.D. \\
\hline Styrene & N.D. & 4.7 & N.D. & N.D. & N.D. & N.D. & N.D. & N.D. & N.D. & N.D. & N.D. \\
\hline m-Xylene & N.D. & 3.3 & N.D. & N.D. & N.D. & N.D. & N.D. & N.D. & N.D. & N.D. & N.D. \\
\hline o-Xylene & N.D. & 7.05 & N.D. & N.D. & N.D. & N.D. & N.D. & N.D. & N.D. & N.D. & N.D. \\
\hline Carbon disulfide & N.D. & 1.9 & N.D. & N.D. & N.D. & N.D. & N.D. & N.D. & N.D. & 21.22 & 132.02 \\
\hline
\end{tabular}

Note: ND = Not Detected. 
Table 6: $\quad$ Comparison of seasonal effluent quality.

\begin{tabular}{|c|c|c|c|c|c|c|c|c|c|c|}
\hline \multirow{2}{*}{ Parameter } & \multirow{2}{*}{$\begin{array}{c}\text { PTARRN } \\
\text { Winter-Spring } \\
2006-2007\end{array}$} & \multirow{2}{*}{$\begin{array}{c}\text { PTARRN } \\
\text { Spring -Summer } \\
2007\end{array}$} & \multirow{2}{*}{$\begin{array}{l}\text { PTARRN Fall- } \\
\text { Winter } 2007\end{array}$} & \multirow{2}{*}{$\begin{array}{l}\text { NOM-127- } \\
\text { SSA1 } 1994\end{array}$} & \multirow{2}{*}{$\begin{array}{l}\text { NOM-001- } \\
\text { ECOL } \\
1996\end{array}$} & \multirow{2}{*}{$\begin{array}{l}\text { Carrizo } \\
\text { Dam }\end{array}$} & \multirow{2}{*}{$\begin{array}{c}\text { Rodri-guez } \\
\text { Dam }\end{array}$} & \multicolumn{3}{|c|}{ Las Palmas Valley } \\
\hline & & & & & & & & Average & Mini-mum & Maxi-mum \\
\hline Cyanide & 0.0138 & 0.0245 & 0.02469 & 0.07 & 1 & NR & NR & NR & NR & NR \\
\hline Free Chloride & 0.79 & 0.042 & 0.0715 & $0.2-1.5$ & NR & NR & NR & NR & NR & $N R$ \\
\hline Chloride & 250.6 & 283.11 & 304.8461 & 250 & NR & 198 & 135 & 767.6 & 121 & 3537.9 \\
\hline $\begin{array}{c}\text { Total } \\
\text { Hardness }\end{array}$ & 760.8 & 488.1667 & 426.923 & 500 & NR & 340 & 224 & 894.9 & 227.6 & 4034.1 \\
\hline Phenols & -0.001 & 0.001 & 0.0025 & 0.001 & NR & NR & NR & NR & NR & NR \\
\hline Fluoride & 1.475 & 1.45 & 0.8353 & 1.5 & NR & NR & NR & NR & NR & NR \\
\hline $\begin{array}{c}\text { Total } \\
\text { Phosphorus }\end{array}$ & NR & 29.935 & 27.5776 & NR & 5 & NR & NR & $N R$ & NR & NR \\
\hline Nitrate & 0.03 & 0.1255 & 1.0507 & 10 & NR & NR & NR & NR & NR & NR \\
\hline Nitrite & 0.012 & 0.0578 & 0.4283 & 0.05 & NR & NR & NR & NR & NR & NR \\
\hline Ammonia & 1.68 & 22.85 & 6.1669 & 0.5 & NR & 0.18 & 0.24 & NR & NR & NR \\
\hline TKN & 21.18 & 41.879 & 14.7115 & NR & 15 & NR & NR & NR & NR & NR \\
\hline $\mathrm{pH}$ & 7.486 & 7.43 & 7.42 & $6.5-8.5$ & $5.0-10.0$ & NR & NR & NR & NR & NR \\
\hline TDS & 1469 & 1318.235 & 1415.4615 & 1000 & NR & 582 & 485 & 2030 & 470 & 7740 \\
\hline Sulfate & 404.8 & 422.8829 & 536.2307 & 400 & NR & 608 & 81 & 274.2 & 43 & 783 \\
\hline Surfactants & 0.2375 & 0.2061 & 0.1761 & 0.5 & NR & NR & NR & NR & NR & NR \\
\hline
\end{tabular}

$\mathrm{NR}=$ Not Reported

\section{Conclusions}

The majority of the effluent's constituents fall below both the maximum levels permitted by the relevant Mexican federal standards and the levels found in the region's major reservoirs. However, if direct reuse is desired, the State Commission of Public Services of Tijuana must plan some type of tertiary treatment.

If the effluent is to be used indirectly for recharging reservoirs by means of infiltration, an acute toxicity study should be done in order to determine the levels at which the effluent would represent a danger to organisms in the receiving body of water. Such a study should be done according to Mexican standard NMX-AA-087-1995-SCFI ${ }^{9}$.

\section{Acknowledgements}

This research has been conducted with the support of Universidad Autónoma of Baja California and assisted by the State Commission of Public Services of Tijuana BC, México

\section{References}

[1] Arreguín F., Moeller G., Escalante V., Rivas A. El reuso del agua en México. Instituto Mexicano de Tecnología del agua. México. 
[2] Cuevas T. Sistema de saneamiento en Tijuana y Playas de Rosarito. Comisión Estatal de Servicios Públicos de Tijuana. 30 agosto 2007.

[3] Wastewater Reuse in the CA region. http://www.dwr.ehnr.state.nc.us/ reports/reuse/reuse.htm

[4] Plan Maestro de Agua Potable y Saneamiento de Tijuana y Playas de Rosarito 2003. Comisión Estatal de Servicios Públicos de Tijuana. 2003.

[5] Northern Rosarito's wastewater treatment plant (PTARRN). (Photo:http://google.earth, 2008)

[6] NMX-AA-003-1995. Aguas Residuales. Muestreo. Diario Oficial de la Nación. México. 25 de marzo 1980.

[7] 8260C, 8270D Test Methods for Organic Compounds. http://www.epa.gov/ epawaste/hazard/testmethods.

[8] NOM-001-ECOL-1996. Que establece los límites máximos permisibles de contaminantes en las descargas de agua residuales en aguas y bienes nacionales. Diario Oficial de la Nación. México. 24 de junio de 1996.

[9] NMX-AA-087-1995-SCFI. Análisis de agua - Evaluación de toxicidad aguda con Daphnia magna Status (Crustacea-Cladecera). Método de prueba. Secretaria de Comercio y Fomento Industrial. 14 noviembre 1995. México. 